\title{
Leveraging the COVID-19 response to improve emergency care systems in the Eastern Mediterranean Region
}

Hani Mowafi, ${ }^{1}$ Hala Sakr, ${ }^{2}$ Hamid Ravaghi, ${ }^{2}$ Osman Elmahal, ${ }^{2}$ Slim Slama, ${ }^{2}$ Dalia Samhouri ${ }^{2}$ and Pryanka Relan ${ }^{3}$

${ }^{1}$ Yale University, New Haven, United States of America; ${ }^{2}$ World Health Organization Regional Office for the Eastern Mediterranean, Cairo, Egypt. 3World Health Organization Headquarters, Geneva, Switzerland. (Correspondence to: Hani Mowafi: hani.mowafi@yale.edu).

Citation: Mowafi H; Sakr H; Ravaghi H; Elmahal O; Slama S; Samhouri D; et al. Leveraging the COVID-19 response to improve emergency care systems in the Eastern Mediterranean Region. East Mediterr Health J. 2020;26(6):626-629 https://doi.org/10.26719/2020.26.6.626

Copyright (C) World Health Organization (WHO) 2020. Open Access. Some rights reserved. This work is available under the CC BY-NC-SA 3.0 IGO license (https://creativecommons.org/licenses/by-nc-sa/3.o/igo).

The COVID-19 pandemic began as a cluster of reported cases of acute respiratory illness in China on 31 December 2019 and went on to spread with exponential growth across the globe. By the time it was characterized as a global pandemic on 11 March 2020 (1), 17 of 22 countries in the Eastern Mediterranean Region (EMR) had reports of infected persons $(2,3)$. EMR countries are particularly susceptible to such outbreaks due to the presence of globally interconnected markets; complex emergencies in more than half of the countries; religious mass gatherings that draw tens of millions of pilgrims annually; and variation in emergency care systems capacity and health systems performance within and between countries (4).

Emergency care is an essential part of the health system and serves as the first point of contact for many around the Region. In times of mass emergency events - including natural disasters, conflict and outbreaks like COVID-19 - the need for care for injuries and other acute conditions increases (5). The emergency care system encompasses key functions from the scene of injury or illness, during transport and through to emergency unit and early inpatient care (6). In well-functioning health systems it is the everyday emergency care system that rapidly surges human, material and organizational resources in times of public health emergency to maintain access to essential emergency care services, to limit direct mortality, and avoid secondary mortality altogether (5).

Even prior to the COVID-19 pandemic, the World Health Organization (WHO) and Member States expressed commitment to scaling up emergency care through resolutions of the Eastern Mediterranean Regional Committee (EM/RC63/R.1) and the World Health Assembly (WHA72.16). In response, WHO at the global and regional levels embarked on initiatives to support ECS development through conducting Emergency Care Systems Assessments in 10 EMR countries to help policymakers coordinate system development activities and use existing processes and resources more effectively. Further work has been done to support implementation of a package of emergency care tools including: the Integrated Interagency Triage Tool (prehospital, routine and mass casualty); Emergency Medical and Trauma Care Checklists; the Basic Emergency Care - an open-access training course for frontline health-care providers who manage acute illness and injury with limited resources; and the International Registry of Trauma and Emergency care to help gather essential data about the performance of emergency care systems $(6,7)$. Despite these positive steps more work is needed to develop and strengthen emergency care systems in the Region. In the coming months many activities that will be undertaken as part of the COVID-19 response will overlap key functions of the emergency care system. These activities should be integrated into the underlying emergency care system to strengthen their ability to maintain access to essential emergency services, and their ability to respond to the current and future public health emergencies.

One important emergency care systems function component is system activation - frequently through an emergency care access number - whereby the public can activate an emergency response in times of illness or injury. Only 13 of 22 EMR countries report having a national emergency care access number (8). Meanwhile, a survey of EMR ministy of health websites, personal communications with ministry of health staff, and reviews of public news reports reveal that 20 of 22 EMR member-states (and Palestine) have established ad hoc national or regional COVID-19 hotlines for the public to access information, report suspected cases, and be screened for symptoms of COVID-19. Early reports indicate widespread utilization of these hotlines with Egypt registering more than 40000 calls per day, prompting the creation of an integrated smartphone application that has been downloaded by over 1 million Egyptians (9).

Overwhelming public demand in the United Arab Emirates for access to COVID-19 information has lead to the creation of a WhatsApp account powered by artificial intelligence to answer the large number of public inquiries. The creation of COVID-19 hotlines and the widespread public use of these numbers present an opportunity to maintain this architecture to create emergency access numbers for system activation in countries where this has been lacking.

Another key functional component is field-tofacility communication. Such pre-arrival notification and coordination between ambulance services and emergency units is rare in the Region (10). Limited availability of personal protective equipment and lack of isolation capability heighten the need to identify 
suspected COVID-19 patients even before their arrival at health facilities. It is essential that as countries establish pre-arrival notification protocols for suspected COVID-19 cases, that they make plans to integrate such field-to-facility communication into routine operations to ensure emergency units are similarly prepared for all critical and time-sensitive cases such as acute myocardial infarction, stroke, and trauma. Doing so can reduce time to treatment and improve outcomes in the Region (11-14).

Despite countries' efforts to control patient flow by directing suspected COVID-19 patients to dedicated facilities, many "self-present" to facilities of their choosing. Moreover, patients presenting for unrelated emergencies (e.g., trauma) may also be co-infected with COVID-19 - whether or not they are symptomatic. It is imperative that all EMR emergency units implement WHO guidance on Clinical Management of COVID-19, including routine screening at the first point of contact with the health system, strict adherence to infection prevention and control measures, and acuity-based triage in order to limit health care worker exposure and ensure that critically ill and injured patients receive timely access to emergency services (15-18).

A recent WHO survey showed that since the COVID-19 pandemic began, $26 \%$ of EMR countries have reported partial or complete disruption of cardiovascular emergency care (19). Updated WHO technical guidance emphasizes management of emergency health conditions, common acute presentations requiring time-sensitive intervention, and 24-hour access to acute care services at first-level hospitals as key elements of maintaining access to essential health services $(20,21)$. EMR countries have begun to explore strategies to maintain access to noncommunicable disease care for populations at highest risk. These efforts should be highlighted to enable the health system to safely meet the overall emergency care needs of the public while preserving facility capacity for the COVID-19 response.

A systematic approach to emergency care - centered on acuity-based triage, early recognition and resuscitation, and simple initial management and referral - has been shown to decrease the mortality associated with a range of medical and surgical conditions (6). Current WHO technical guidance not only includes systematic approaches to Clinical Management of COVID-19 but also includes the Basic Emergency Care Course as essential guidance for management of suspected COVID-19 cases, as well as the larger population, which continues to present with acute conditions and excerbations of chronic illness. EMR countries should re-double efforts to train frontline providers in Basic Emergency Care, as these systematic approaches to emergency care are crucial to saving lives now and in the future.

Throughout the Region, populations displaced by conflict and social dislocation are settled in camps and low-income communities characterized by close quarters and inadequate access to water and sanitation needed to manage an infectious disease outbreak. Additionally, many EMR countries lack legislation guaranteeing access to emergency care for all (a key WHO Health System Building Block under governance), which limits access to marginal communities (10). Since the Region is host to the largest number of displaced persons in the world, region-specific guidance has been developed to guide health system response to COVID-19 in the context of displacement (22). What is further needed, however, is the rationalization of health policies to ensure that all acutely ill or injured persons may access emergency care to limit unnecessary morbidity and mortality and to eliminate potential reservoirs of infection in the wider community.

Violence against health care workers has increased globally in the context of COVID-19 (23). Such workplace violence is unfortunately routinely prevalent in emergency units across the Region and frequency of exposure to violence is cited as a major cause of health worker burnout in EMR countries (24-28). Ensuring patient and provider safety are core cross-cutting functional components of the WHO Emergency Care Systems Framework. It is imperative that EMR countries not only implement WHO guidance on rights, roles and responsibilities of health workers to include increased security for emergency units and ambulance teams, but that they support a zero-tolerance policy towards violence against health workers and establish monitoring and reporting of violations to protect emergency patients and staff (29).

Lastly, there is a paucity of high-quality published data on emergency care systems in the Region and an urgent need for operational research to understand the emergency care needs and emergency care systems performance in EMR countries. This is also true in the context of COVID-19, with only a few published reports of primarily single facility experience rather than systematic analysis of the emergency care system performance in this time of public health emergency. Establishing robust emergency care data collection through the International Registry of Trauma and Emergency Care, and linking to other existing public health information systems, can strengthen emergency surveillance systems. In addition, it can serve as a bridge between public health emergency response and ongoing quality and performance improvement of national emergency care systems (30).

The COVID-19 pandemic represents a stress test of countries' health systems. Policy-makers in the Region must seize the opportunity to identify gaps in their emergency care systems, thereby integrating elements of the pandemic response to create lasting improvements that strengthen the ability of their emergency care system to respond to routine and public health emergencies in the future. 


\section{Reference}

1. World Health Organization. WHO Director-General's opening remarks at the media briefing on COVID-19. Geneva: World Health Organization; 11 March 2020. (https://www.who.int/dg/speeches/detail/who-director-general-s-opening-remarks-at-themedia-briefing-on-covid-19---11-march-2020, accessed 26 March 2020).

2. World Health Organization Regional Office for the Eastern Mediterranean (WHO/EMRO). Eastern Mediterranean Region COVID-19 Affected Countries. Cairo: WHO/EMRO; 2020. (https://app.powerbi.com/view?r=eyJrIjoiN2ExNWI3ZGQtZDk3MyooYzE2LWFjYmQtNGMwZjkoOWQ1MjFhIiwidCI6ImY2MTBjMGI3LWJkMjQtNGIzOSo4MTBiLTNkYzI4MGFmYjU5MCIsImMiOjh9, accessed 26 March 2020).

3. World Health Organization Regional Office for the Eastern Mediterranean (WHO/EMRO). Coronavirus disease 2019 (COVID-19) Weekly Situation report 02. Cairo: WHO/EMRO; 2020.

4. World Health Organization. WHO Eastern Mediterranean Region: Joint External Evaluation Mission Reports. Geneva: World Health Organization; 2005 .

5. World Health Organization. Emergency Care System Framework. Geneva: World Health Organization; 2018. (https://www.who. int/publications-detail-redirect/who-emergency-care-system-framework, accessed 17 June 2020).

6. Reynolds T, Sawe H, Rubiano A, Shin SD, Wallis L, Mock CN. Strengthening health systems to provide emergency care. In: Jamison DT, Gelband H, Horton S, Jha P, Laxminarayan R, Mock CN, Nugent R (ed). Disease control priorities: improving health and reducing poverty. Washington, DC: World Bank; 2018:247-65.

7. World Health Organization. Emergency Care. Geneva: World Health Organization; 2020. (https://www.who.int/health-topics/ emergency-care\#tab=tab_2, accessed 26 March 2020).

8. World Health Organization. Global status report on road safety 2018. Geneva: World Health Organization; 2018.

9. $\quad$ Over a million people download Egypt's coronavirus phone app. Ahram Online. 31 May 2020.

10. World Health Organization Regional Office for the Eastern Mediterranean (WHO/EMRO). Emergency Care as an essential component of Universal Health Coverage. 63rd Session of the WHO Regional Committee for the Eastern Mediterranean. Cairo: WHO/EMRO; 2016.

11. Ting HH, Krumholz HM, Bradley EH, Cone DC, Curtis JP, Drew BJ, et al. Implementation and integration of prehospital ECGs into systems of care for acute coronary syndrome. Circulation 2008;118(10):1066-79.

12. Klein K, Lefering R, Jungbluth P, Lendemans S, Hussmann B. Is prehospital time important for the treatment of severely injured patients? A matched-triplet analysis of 13,851 patients from the TraumaRegister DGU ${ }^{\circledR}$. BioMed Res Int. 2019:5936345.

13. Liberman M, Mulder DS, Jurkovich GJ, Sampalis JS. The association between trauma system and trauma center components and outcome in a mature regionalized trauma system. Surgery 2005;137(6):647-58.

14. Sheppard JP, Mellor RM, Greenfield S, Mant J, Quinn T, Sandler D, et al. The association between prehospital care and in-hospital treatment decisions in acute stroke: a cohort study. Emerg Med J 2015;32(2):93-9.

15. Casagranda I, Coen D, Paolillo C, Cervellin G, Bellone A, Perlini S, et al. Changing Emergency Department and hospital organization in response to a changing epidemic. Emerg Care J. 2020;16(1). https://doi.org/10.4081/ecj.2020.8969

16. Qing MX, Li S, Yu S, Ouyang Y, Zeng L, Li X, et al. Emergency management of the prevention and control of novel coronavirus pneumonia in specialized branches of hospital. Acad Emerg Med 9 March,2020; https://doi.org/10.1111/acem.13958

17. World Health Organization. COVID-19: Strategic planning and operational guidance for maintaining essential health services during an outbreak. Geneva: World Health Organization; 2020.

18. Zeng J, Huang J, Pan L. How to balance acute myocardial infarction and COVID-19: the protocols from Sichuan Provincial People's Hospital. Intens Care Med 2020 Jun;46(6):1111-1113

19. World Health Organization. Rapid assessment of service delivery for NCDs during COVID-19 pandemic. Geneva: World Health Organization; 2020.

20. World Health Organization. Maintaining essential health services: operational guidance for the COVID-19 context: Interim Guidance 01 June 2020. Geneva: World Health Organization; 2020.

21. World Health Organization. Pillar 9: Maintaining essential health services and systems. Geneva: World Health Organization; 2020. (https://covid-19-response.org/pillar/9, accessed 14 June 2020).

22. World Health Organization Regional Office for the Eastern Mediterranean (WHO/EMRO). WHO interim guidance: Health system response to COVID-19 in the context of internally displaced persons, refugees, migrants and returnees in the Eastern Mediterranean Region. Cairo: WHO/EMRO; 2020.

23. McKay D, Heisler M, Mishori R, Catton H, Kloiber O. Attacks against health-care personnel must stop, especially as the world fights COVID-19. Lancet 2020;395(10239):1743-5.

24. AlBashtawy M, Al-Azzam M, Rawashda A, Batiha A-M, Bashaireh I, Sulaiman M. Workplace violence toward emergency department staff in Jordanian hospitals: a cross-sectional study. J Nurs Res 2015;23(1):75-81.

25. Abdellah RF, Salama KM. Prevalence and risk factors of workplace violence against health care workers in emergency department in Ismailia, Egypt. Pan Afr Med J 2017; 26(1). 
26. Nayyer ul I, Yousuful-Islam M, Farooq MS, Mazharuddin SM, Hussain SA, Umair ul I. Workplace violence experienced by doctors working in government hospitals of Karachi. J Coll Physicians Surg Pak 2014;24(9):698-9.

27. Abdo SA, El-Sallamy RM, El-Sherbiny AA, Kabbash IA. Burnout among physicians and nursing staff working in the emergency hospital of Tanta University, Egypt. East Mediterr Health J 2016;21(12):906-15.

28. Harthi MM, Olayan MA, Abugad HA, Wahab MMA. Workplace violence among health care workers in emergency departments of public hospitals in Dammam, Saudi Arabia. East Mediterr Health J. 2020;26(x):xxx-xxx http://doi.org/10.26719/emhj.20.069 [In Press]

29. World Health Organization. Coronavirus Disease (COVID-19) outbreak: rights, roles and responsibilities of health workers, including key considerations for occupational safety and health. Geneva: World Health Organization; 2020.

30. Mowafi H, Ngaruiya C, O’Reilly G, Kobusingye O, Kapil V, Rubiano AM, et al. Emergency care surveillance and emergency care registries in low-income and middle-income countries: conceptual challenges and future directions for research. BMJ Glob Health 2019;4(Suppl 6):e001442. 